\title{
A network based methodology to reveal patterns in knowledge transfer
}

\author{
Orlando López-Cruz, Nelson Obregón N. \\ ${ }^{1}$ Universidad El Bosque, Bogotá, \\ ${ }^{2}$ Universidad Javeriana, Bogotá
}

\begin{abstract}
This paper motivates, presents and demonstrates in use a methodology based in complex network analysis to support research aimed at identification of sources in the process of knowledge transfer at the interorganizational level. The importance of this methodology is that it states a unified model to reveal knowledge sharing patterns and to compare results from multiple researches on data from different periods of time and different sectors of the economy. This methodology does not address the underlying statistical processes. To do this, national statistics departments (NSD) provide documents and tools at their websites. But this proposal provides a guide to model information inferences gathered from data processing revealing links between sources and recipients of knowledge being transferred and that the recipient detects as main source to new knowledge creation. Some national statistics departments set as objective for these surveys the characterization of innovation dynamics in firms and to analyze the use of public support instruments. From this characterization scholars conduct different researches. Measures of dimensions of the network composed by manufacturing firms and other organizations conform the base to inquiry the structure that emerges from taking ideas from other organizations to incept innovations. These two sets of data are actors of a twomode-network. The link between two actors (network nodes, one acting as the source of the idea. The second one acting as the destination) comes from organizations or events organized by organizations that "provide" ideas to other group of firms. The resulting demonstrated design satisfies the objective of being a methodological model to identify sources in knowledge transfer of knowledge effectively used in innovation.
\end{abstract}

Keywords - Knowledge Transfer; Technological Innovation; Technology Transfer; Social Networks Analysis.

\section{INTRODUCTION}

$\mathrm{T}$ HIS paper is intended to introduce and show the application of a methodology to identify the underlying structure of the process of knowledge transfer at the inter-organizational level, as one of the main resources to create innovation.

Even the search for methods to understand and design effective processes to incept innovation is an active thread in contemporary research, the dominant research paradigms used to inquiry on knowledge and technology transfer (TT) continue to be those traditional descriptive research methods of the natural and social sciences.

The strength of the proposed methodology is its simplicity. Even that, it seeks to provide a unified model to reveal knowledge sharing patterns about different periods of time and different sectors of the economy.

This methodology complements the statistical methodology provided by the NSD on SITD and SITDS surveys, by unify the way to identify sources and recipients links when knowledge is needed to be transferred procuring to generate innovation in firms.

To present the results of the application of the proposed methodology, this paper is structured as follows: Section II introduces the concepts of knowledge and knowledge sharing. Section III describes SITD and SITDS as the origin of raw data to search for structures of knowledge transfer. Section IV explains the reason why when investigating on technology transfer is unavoidable to address the technological knowledge transfer topic. Section V introduces a categorization of technology transfer models which resulted from a literature review.

In section VI, the mathematical foundations for representation and analysis of social networks are introduced. Sections VII and VIII describe the proposed methodology to be demonstrated in use. Section IX shows the case of the manufacturing sector where the proposed methodology is demonstrated in use. The results are shown in Section $\mathrm{X}$, and discussion and conclusion are presented in sections XI and XII. Finally, the references used in the preparation of the paper are listed.

\section{NATURe OF KNOWledge Sharing}

\section{A. Knowledge}

It is understood that when innovation is a desirable outcome, knowledge is a critical supply to organizations [1, 2]. Knowledge is a multidimensional concept that has been studied since ancient Greeks, and becomes relevant in modern organizations specially when data processing and information systems have not explained nor commanded organizations viability. The role of knowledge in organizations emerged as a key concept over data and information [3].

Under the Shannon and Weaver's communication paradigm [4], there are three levels of communication complexity: syntactic, semantic and pragmatic. These allow to describe knowledge across three organization boundaries: information- processing boundary at a syntax level, interpretive boundary, and pragmatic boundary [5]. These, in turn, allow transferring, translating and transforming knowledge [5]. These determines a structure composed by the link between organizations sharing knowledge and organization.

In this context, knowledge is shared at three different non-exclusive levels. While data are conceived as a set of facts or a symbolic record of facts, without interpretation, information is understood as those data in a context with a sender and a receiver [6]. When available information conducts to both comprehension and action, on the context of that information, emerges knowledge [7]. Knowledge is not tradable (i.e. knowledge may not be subject of a sale transaction), therefore it must be "thought" [8] and learned.

\section{B. Knowledge sharing}

In this work, knowledge sharing is the act by which humans share knowledge in a community or set of organizations [8]. This does not imply nothing about the intention to get economic benefits or satisfy 
individual interests.

In this context, knowledge transfer, is a possibility when sharing knowledge, but it depends on conditions of the context where knowledge appears, conditions of the context where may appear, and a possible link between those two contexts.

\section{DATA TO SEARCH FOR SHARING STRUCTURES}

In order to search for structures enabling knowledge sharing, raw data gathered by the National Department of Ststistics of Colombia (NDS) was used. This Department is the head of the National Statistics System which is integrated by governmental organizations and autonomous organizations, according to Colombia national regulations and laws.

By 1996, the NDS conducted the first national survey on technological development (STD). It was conducted on firms of the manufacturing sector of the economy (Fig.1). The second survey was conducted nine years later, in 2005.

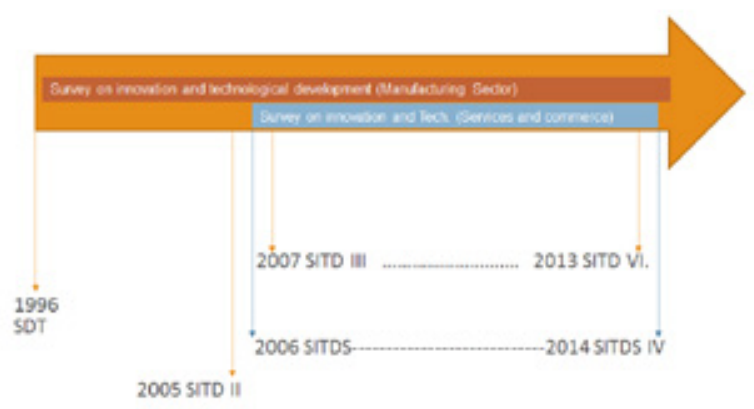

Fig. 1. Timeline showing the years when the survey on innovation and development technology for the manufacturing sector (SIDT) and the corresponding survey for the services and commerce sector (SITDS) has been conducted. The very first SITD was just a survey on development technology (SDT) in 1996. SITD and SITDS are conducted every two years. SITD in odd years and SITDS in even years.

Since 2005, the survey is known as the survey on innovation and technological development (SITD) and is being conducted every odd year and data gathered correspond to the two preceding years. For instance, SITD-2015 gathers data from 2013 and 2014.

SITD is characterized by a survey on the manufacturing sector of the economy. Therefore, since 2006 the NDS conducts an additional survey to gather data from the sector of services and commerce. The national survey on innovation and technological development-services (SITDS), conducted by NDS every even year since 2006, gathers data corresponding to two years preceding the survey. This means that SITDS-2014 is composed by data from 2012 and 2013.

SITD as well as SITDS aim for a (statistically) characterization of innovation dynamics of firms. In addition, both of them aim for an analysis of the usage of public instruments at the production and services sectors of the economy.

\section{ECONOMIC DEVELOPMENT: FROM TECHNOLOGY TRANSFER TO KNOWLEDGE TRANSFER}

In economics, "development" refers to the process of improvement of the economic, political, and social well-being of people [9-12]. Not all societies sustains the same development rhythm and economies experiment cyclic behaviors [13-15]. This leads to different levels or stages of economic development of countries.

In order to continue catching up with developed countries, less developed countries import technology. In turn, organizations in recipient countries face the challenge to incorporate imported technology to their production processes in order to increase productivity.

But, even though technology were a commodity, transference is not a gentle process $[16,17]$. First, recipients must choose where to take technology from or where to get ideas to improve their processes. Then, find out the way to incorporate technology to the production processes. In this way, TT needs to be more than movement of physical assets.

Accepting that TT is the process by which commercial technology, a technological innovation and the related knowledge is disseminated $[18,19]$, then the issue is what is understood as technology.

Technology include tools, techniques, materials and power resources developed by humans to achieve their goals [20, 21]. Technology may include physical artifacts, but ever includes knowledge [21]. TT is valuable to increase productivity in so far as transferred knowledge allows to modify production processes. This has been an issue for economists [22] but on a retrospective or an explanatory point of view.

\section{Technological KNOWLEdge Transfer CATEgories}

\section{A. Technological knowledge transfer}

Transferring technology is not achieved without transferring associated knowledge [23]. Knowledge most not be reified, this means that knowledge should not be understood as an object outside individuals and their environment as a historical and cultural context. Knowledge is different form information and data: knowledge is a human act [8] related to a human process inside human being's mind [24], therefore, knowledge appears at the orbit of personal thoughts and experiences [25, 26].

Technological artifacts are not just the physical object. Besides any material instantiation of technology, they include mental models [8] that make sense the usage of the artifact by a community. Consequently, any TT must be thought as knowledge transfer also.

\section{B. A categorization of technological knowledge transfer}

Some authors introduce categories of TT. A four-categories model [27] of TT, according to strengths, limitations and focusing consists of 1) a category of traditional models, composed by appropriability, dissemination, and communication models, 2) 1990's models including the Gibson and Smilor model [28], Sung and Gibson model [29], and Rebentish and Ferreti model [30], 3) Knowledge-based model [27, 31, 32], and 4) organizational learning models [33] (Table I). The last two categories may be thought as two sides of the same coin. However, in this paper are set apart.

Traditional models are reductionist (Table I). In the appropriability model, the technology developer assumes a passive role: quality technologies sell themselves. It is linear because states as in imperative for research the production of technological developments that are realized in the market.

The dissemination model focuses on diffusion [19]. In this model, knowledge freely flows from the expert to the non-expert user. It is supposed that a researcher lacks of prejudices to disclose his innovations and the potential user is eager to know innovations. Linearity comes from the unidirectional communication to the user, who appears to be aligned at the end of the TT process as "final user".

In contrast, the usage model of knowledge includes a three.way communication between researcher, developer and user of the technology. This model does not explain TT beyond organization boundaries.

The communication model perceives TT as an on-going process where individuals share ideas. This model follows the network 
paradigm where feedback is ubiquitous. Knowledge in this model is understood as an independent object, universally applicable, similar to scientific knowledge.

Models of TT in the 1990's category (Table I) are focused to solve limitations in traditional models. In the Gibson and Smilor model [28] TT is a passive process defined by three levels of involvement, from the relationship researcher-user point of view: (I) technology development, (II) technology acceptance, and (III) technology application. It emphasizes in level I, taking market pressures and research quality causing TT. This makes this model similar to the appropriability model.

TABLE I

CATEGORIES FOR TECHNOLOGICAL KNOWLEDGE TRANSFER MODELS a

\begin{tabular}{ccc}
\hline Category & Model & Time \\
Traditional & Appropriability & $1945-1950$ \\
& Dissemination & $1960-1970$ \\
& Knowledge usage & 1980 's \\
& Communication & $1980-1991$ \\
1990's & Gibson \& Smilor & 1991 \\
& Rebentisch \& Ferreti & 1995 \\
& Sung \& Gibson & 2000 \\
$\begin{array}{c}\text { Knowledge-based vision } \\
\text { Organizational-Learning } \\
\text { based vision }\end{array}$ & KBV & $1985-2000$ \\
\hline \hline
\end{tabular}

a. Possible models for technological transfer appeared before the 20th century are not included in this table.

The Sung and Gibson model [29] is an enhancement of the Gibson and Smilor model [28] to four levels: (I) Creating knowledge and technology (II) deployment, (III) Implementation, and (IV) Commercialization. Knowledge created at level I spreads through publications, teleconferences, and massive media, not including the user. Therefore, this is a passive process.

The Rebentish and Ferreti model [30] is developed from the point of view of the transferor. It emphasizes knowledge embodied in assets being transferred (explicit knowledge [34, 35]). Even though its focus on the relationship between technology-organizational context, and the organization capacity to adopt new technology, the model is linear biased because omits actors of the TT process.

The Knowledge-Based View (KBV) [2, 36] of TT (Table I) understands firms as sets of knowledge and knowledge in firms produce competitive advantage. The aim is to get a sustainable advantage by means of knowledge. This view conceives knowledge as an intangible resource, hardly transferable on an intra-firm basis. A firm may be modeled as an instrument to transfer and develop knowledge next to other related organizations, not just as a knowledge repository. The model acknowledges insight and individual abilities as (tacit) knowledge that is difficult to assemble and transfer, but that allows to bring together operational issues in learning [37 p.430].

Organizational Learning (OL) [33] (1) as a cognitive process with and without intention, sets up links between actions in the past and in the future, forming a stock of organizational knowledge and memory. (2) as a change in behavior and improvement in organizational effectiveness, make changes operative through modifications in individual, group and firm behavior, and (3) both as cognitive process and change in organizational behavior, OL allows behavior changes to improve organizational performance and to develop new knowledge.

As stated before, KBV and OL are two sides of the same coin. KBV and $\mathrm{OL}$ act as a knowledge acquiring unit and as an individual, group and firm behavior modification unit.

\section{REPRESENTATION OF SOCIAL NETWORKS}

The technical analysis conducted in this study is based in network analysis, a discipline which stands on the innovation of Jacobo Levy Moreno: the sociogram [38]. This was applied in the measurement of the interpersonal relations in small groups, known as sociometry [39] since 1934 [40]. The relations are studied using graph theory. This allows to represent, design, and calculate properties on networks

\begin{tabular}{l|l|l|l|l} 
& $\mathrm{m} 1$ & $\mathrm{~m} 2$ & $\mathrm{~m} 3$ & $\ldots$ \\
\hline $\mathrm{n} 1$ & \multicolumn{2}{|c|}{$\mathrm{N} \times \mathrm{M}$} & & \\
\hline $\mathrm{n} 2$ & \multicolumn{2}{|c|}{$\mid$} & & \\
\hline $\mathrm{n} 3$ & & &
\end{tabular}

Fig. 2. A two.mode network where the set of nodes $n 1, n 2, n 3, \ldots$ may represent the source of information or "ideas" for innovation and the set of nodes $\mathrm{m} 1, \mathrm{~m} 2, \mathrm{~m} 3, \ldots$ the destination of those information and "ideas".

[41].

A network (a graph) may be understood in its basics as a collection of points (nodes) joined together in pairs by lines (edges). To design a network model [42] the starting point is the measures of the properties of the network (i.e. properties of the phenomenon to be studied or the state to achieve), for instance: number of edges, number of nodes, degree of vertexes, and clustering coefficient. There are many other properties of networks that may be inferred (calculated) [43]. This model is not a single network, but a probability distribution on many networks (i.e. an assemble model).

If the network is to be designed to comply with some characteristics, generative network models are to be used [41]. In the many generative network models, the preferential attachment models seems to be the most adequate to design the growth of complex networks exhibiting power laws. Preferential attachment models may be the Price model, the Barabási and Albert model, the vertexes copying model, and network optimization model.

Newman [41] affirms that Price was inspired by H.A.Simon works [44] In his paper, Simon does not name the distributions, but Price name them as cumulative advantage distributions, that describe the St. Matthew principle (Mt. 25:29).

\section{A. A mathematical representation of a social network data}

Social network data is determined by the substantive concern or theories that support the specific study of a network, and is composed by at least one structural variable of a dataset of the phenomena or field in study [40].

The network under study may be composed by one or more datasets, according to the nature of the data (i.e. the different kind of social network entities involved in the study). The number of different datasets composing data of the network is said to be the "mode" of the network [40]. In this study, there are two distinguishable sets of entities: organizations acting as receivers of information and "ideas" conducting to innovation, and organizations acting as source of those information and "ideas" (Table II). These two sets conform actors of the network that are related each other by means of the link of source/ destination of that information.

Appears a two-mode (or 2-mode) network in which clearly there are directed relations (source/destination) (Fig. 2). 
TABLE II

EXTERNAL SOURCES OF IDEAS GENERATING INNOVATION

\begin{tabular}{cc} 
No. & Source of ideas a \\
1 & Professional associations or sectorial associations. \\
2 & Scientific and technological databases. \\
3 & Chambers of Commerce. \\
4 & Technological development center. \\
5 & SENA training center. \\
6 & Research centers. \\
7 & Regional productivity center. \\
8 & Clients. \\
9 & Competitor or firm from the same economical sector. \\
10 & Consultants or experts. \\
11 & R\&D departments of other firms. \\
12 & Firm from other economic sector. \\
13 & Trade fairs and exhibitions \\
14 & Technology-based companies and incubators. \\
15 & Public institutions. \\
16 & Internet. \\
17 & Industrial property information systems. \\
18 & Universities. \\
19 & Technical regulations and standards. \\
20 & Technological parks. \\
21 & Suppliers. \\
22 & Seminars and conferences. \\
23 & Copyright information systems. \\
\hline &
\end{tabular}

Previous order is not relevant and does not bias the analysis.

${ }^{a}$ Lexicographically ordered in the original in Spanish.

The intersection of rows and columns in the matrix (Fig. 2) may represent the strength of the relation between source and destination or just a binary value to represent if there exist (1) or not (0) a relation between nodes $n_{i}$ and $m_{j}$. This is the case in this study where the aim is to identify the sources of knowledge for innovation that some organizations use.

\section{B. Patterns of links because of knowledge sharing}

But it is not statistical data the interest of this study but the structures for innovation in an economical sector. Therefore, patterns are to be discovered from data. In order to do that, a concept capturing "where knowledge comes from" is needed.

The concept is the degree of the node. Since the relation represents source/destination of information and "ideas" for innovation, then the number of edges over a node may represent the importance of a node. Since the degree of the node $d\left(n_{i}\right)$ is the number of edges incident to node $n_{i}$, when measuring nodal degree for each of the nodes in the network, a pattern will appear.

If $\mathrm{X}$ is the sociomatrix of a two-mode network, $\mathrm{x}_{\mathrm{ij}}$ is an entry of that matrix, and $g$ is the total nodes of the directed-graph of a network, the number of edges incident to node $n_{i}$ from nodes $n_{j}$ is defined as the indegree $\left(\mathrm{d}_{\mathrm{I}}\right)$ of the $i$-esim node $\left(\mathrm{n}_{\mathrm{i}}\right)$, and may be calculated as $\mathrm{d}_{\mathrm{I}}\left(\mathrm{n}_{\mathrm{i}}\right)$ according to (1):

$$
d_{I}\left(n_{i}\right)=\sum_{j=1}^{g} x_{j}
$$

The higher the value of $d_{i}\left(n_{i}\right)$ the node $n_{i}$ is more "important" in the network. In this case, node $n_{i}$ stands as a relevant source of knowledge in a network (of organizations).

\section{The problem to be solved}

Up to this point, there seems no problem to be solved. Raw data on the relationships between organizations in an economy is provided by a national survey on manufacturing, services and commerce sectors of the economy. A wide theory on inter-organizational knowledge sharing is being developed, and, graph theory provides a mathematical representation of networks and its properties.

However, looking closely to the task to discover patterns of the relationship between organizations needing to innovate and those providing "ideas" for innovation along time, and the process of understanding the complex innovation path followed by organizations in an economy, there are no way to compare the results of different studies, since every researcher develops an ad-hoc methodology group [45] or develops a complicated methodology that prevents its usage [46], which reinforces the trend to use specific methodologies. This will not be a problem unless results need to be compared by the industry to make decisions.

Therefore, both industry and researchers lack of an easy to understand and apply, but powerful methodology, to reveal knowledge flows that produce innovations. This proposal intends to fill this gap.

VII. A METHODOLOGY TO REVEAL PATTERNS IN KNOWLEDGE
TRANSFER

\section{A. What 'methodology'means}

In simple terms, a methodology is "a system of principles, practices, and procedures applied to a specific branch of knowledge" [47]. From a process view, a methodology is a device specifying a set of steps and restrictions on the transitions between those steps $[48,49]$. In addition, a methodology may be understood as the means that conducts to produce the solution to a problem [50]. In the context of this study, a methodology is an artifact that consists of a list of procedures, each one acting stepping stones, the conditions to proceed between procedures, in order to solve a problem. This artifact may be provided to different researchers, in the same context, to produce consistent results.

\section{$B$. The relevance of "patterns" in (research) methodologies}

Patterns have been present in human knowledge since ancient Greeks. In Euclid's Elements, geometric constructions play the role of patterns in mathematical reasoning. Besides, this geometrical patterns leverages reasoning processes to solve problems.

According to these, any problem is identified by three components. (a) In any problem there must be a thing required or desired (the unknown). Without an unknown, there is nothing to look for, here is nothing to seek [51]. In our research problem the unknown is the structure of relations between sources of ideas for innovation and innovators. In addition, (b) in any problem there must be something given. Without any given (known) that serves as a reference, there is nothing by which the required thing may be recognized [51]. In our research problem, 'data' are SITD and SITDS surveys raw data. This is required because even if we see the required thing we couldn't recognize it. And, finally, (c) in any problem there must be a condition which specifies how the unknown is linked to the given data [51].

The procedure that has been describe above is a 'methodology' to solve (mathematical) problems. The key concerns is that it is, in itself, a pattern. Something that may be imitated even tackling different problems of the same class. 
It should be noted that the patterns is not (necessarily) a function. There is no 'parameter' passing. Further, it is not a (simple) procedure that, as a recipe, may be indefinitely repeated giving the same results. It is not a blind computational routine.

It is closer to scripts [52] in the sense that describes the purposes of every role, but the actual staging results in different instances of the same play. In project management, but in a special sense, in software project management projects, it is clear that even when a project manager follows a software development methodology, the manager can never attend the same script in the same way [52] to carry a project out. Nothing guarantees success. However, the pattern is relevant to improve the chances of success.

The structural part of processes, even under changing conditions, preserves the identity of the process itself. Each time a software process is conducted it becomes a new process [53], which recalls for the dynamics of adaptive systems. A short-term gentle adjustment of the structure to an evolutionary environment makes to evolve (coevolve) the whole that is being studied, either the whole is a software development process $[52,54]$ or a technology transfer process $[28,29$, 31] there exist a structure revealed by patterns [55].

The following sections introduce the procedures of the methodology and the rules to proceed between them.

\section{Obtaining data from surveys}

Select the periods of time corresponding to the elapsed time of interest. Given that SITD and SITS are available since 2005 and 2006, respectively, including data corresponding to the preceeding two years, on a two-year basis, the researcher should adjust the research window. The public character of data of the NDS guarantee availability in plain format or spreadsheet format.

\section{ISIC standardization}

When the study includes data from different years for SITD or SITDS, the researcher should make sure that data is classified according to compatible revisions of the International Standard Industrial Classification of All Economic Activities (ISIC). Not all categories of ISIC persists between periods. The researcher should be aware of changes in definitions of each code between revisions (for instance between ISIC Rev 3 and ISIC Rev 4. New codes implies to define dummy codes when constructing a sociomatrix for a previous year.

\section{E. Define dimensions and datasets}

Identify the dataset of recipient and the dataset of source of ideas. The source may be further classified according to the boundary of the recipient organization. Blurry boundaries should be documented to state clearly the meaning of (i) internal source of ideas, (ii) external source of ideas. This last category may be even further classified in: other firms, specialized groups, and external relationships).

Define as 'dimension 1' all actors (and its data), corresponding to internal sources. Keep ISIC classification in the following categories: (a) Internal R\&D departments, (b) production department, (c) Sales and marketing department, (d) other department of the firm, (e) interdisciplinary groups, (f) management staff, and (g) workers.

As 'dimension 2' include other firms. Keeping ISIC classification of data, the categories are: (a) external R\&D department, (b) other related firm, (c) headquarters, (d) clients, (e) competitors, and (f) suppliers.

'Dimension 3', specialized groups, is composed by (a) professional associations or sectorial associations, (b) Chambers of Commerce, (c) agricultural and forest research centers, and (d) technological development center.

Set 'Dimension 4', external relations, as (a) SENA training centers, (b) Consultants and experts, (c) trade fairs and exhibitions, (d) seminars, (e) Books, journals and catalogs, (f) Intellectual property information systems, (g) copyright information systems, (h) Internet and other ICT, and (i) scientific databases.

The first dataset is the matrix resulting from the cross-match between dimension 1 and ISIC. These represent actors of a 2-mode network.

The second dataset is the matrix resulting from the cross-match between dimensions 2, 3 and 4 and ISIC. These represent actors of a 2-mode network.

\section{SOCIAL NETWORK DATA PROCESSING}

Input datasets into a computer software specialized in network analysis. Software packages often provides a data import utility to accomplish this cumbersome time-consuming activity. Software may be one of the following: UCINET+NetDraw, Egonet, Gephi, Pajek, iGraph, JUNG, Statnet.

Apply the projection operator selecting first dimension 1, and then dimensions 2, 3, and 4. This is to perform an oriented analysis to determine relevant sources (rows). If possible, select $\mathrm{MCO}$ minimum square method, as a valued (non-binary) network. The co-occurrence method is adequate for binary networks.

Then, calculate eigenvalues by Single Value Decomposition SVD). This allows to identify background dimensions of the space (set) sector-by-source of the idea.

Finally, group by degree centrality. For each node of te network calculate degree centrality and graph.

\section{CASE OF THE MANUFACTURING SECTOR}

In order to study technological knowledge transfer to industrial firms in the manufacturing sector of the Colombian economy, and determine the structure (main links between sources of ideas of innovations and firms) the proposed methodology was applied.

It is assumed that TT is verified when an incoming idea produces a technological innovation in the recipient firm. Data from SITD-IV serve as data to explore the relations between sources/destination of ideas for innovations. External sources are grouped in twenty four (24) categories (Table II).

Besides, recipient organizations -the organizations producing innovations from incoming ideas- are grouped in 64 subsectors by ISIC Rev.3 (Rev.3 A.C.).

Raw data was classified (rows) according to firm size (small, medium and large enterprise, and then populated a matrix. Columns were sorted buy ISIC Rev.3 code sector. A further classification allowed to set national and foreign firms apart.

The result, each of eight matrixes describes a two-mode network, then they were transformed from a 2-mode network by means of a projection method. This was done by selecrting on of the two datasets and linking the nodes according to the rule: it is connected with another node of the other set. It was used [56], projecting a two-mode network on a weighed one-mode network to preserve the structure of the original network. The networks were drawn [57].

\section{Results}

Data from social networks were analyzed and organized in relatively homogeneous networks according to national or foreign origin of the firm that originates the idea.

The analysis proceeds by the size of the firm: large, medium or small, keeping the origin (national or foreign origin) of the firm. Fig. 3 shows the 2-mode network for large firms taking ideas from foreign sources. 


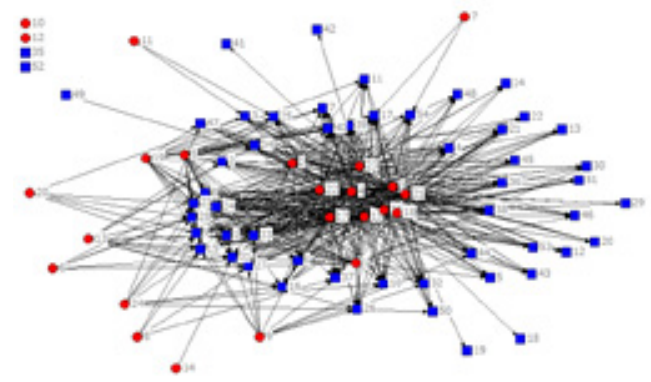

Fig. 3. Two-mode network for large firms with ideas from foreign sources.

Red nodes correspond to the dataset of source of ideas, while blue nodes correspond to the second dataset: manufacturing sector firm category (ISIC). Resulting data shows that sources of higher centrality are as follows (according to their indegree): 1 (151), 2 (152), 3 (153), 4 (154), 15 (175), 16 (181), 17 (191), 18 (192), 21 (203), 22 (210), and 23 (221), as foreign sources for large firms. It is remarkable that foreign sources 10 (160), and 12 (172) are not relevant sources for large firms. Furthermore, firms in subsectors 35 (281) y 52 (359) do not use foreign sources of ideas to incept innovation.

The same conventions apply for the two-mode network of large firms and national sources of ideas (Fig. 4).

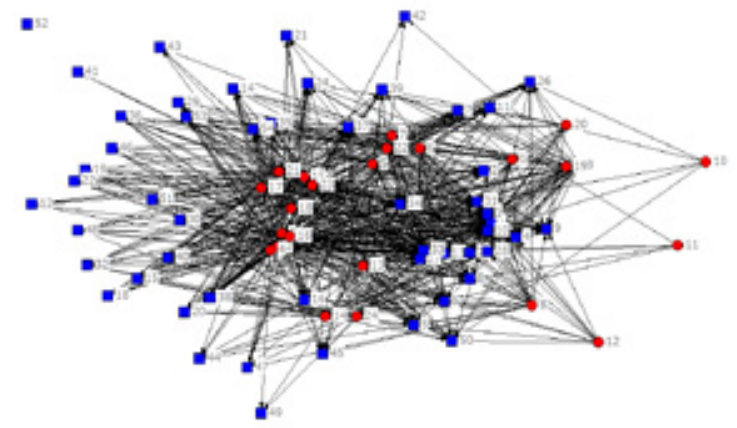

Fig. 4. Two-mode network for large firms with ideas from national sources.

Similarly, to the previous analysis, sources of higher centrality are: 2 (152), 4 (154), 6 (156), 7 (157), 13 (173), 15 (175), 16 (181), 17 (191), 18 (192), 21 (203), 22 (210), and 23 (221).

The case for medium enterprises receiving ideas from foreign sources (Fig. 5) reveal the higher centrality as follows: 2 (152), 3 (153), 4 (154), 16 (181), 18 (192), 21 (203), 22 (210) and 23 (221).

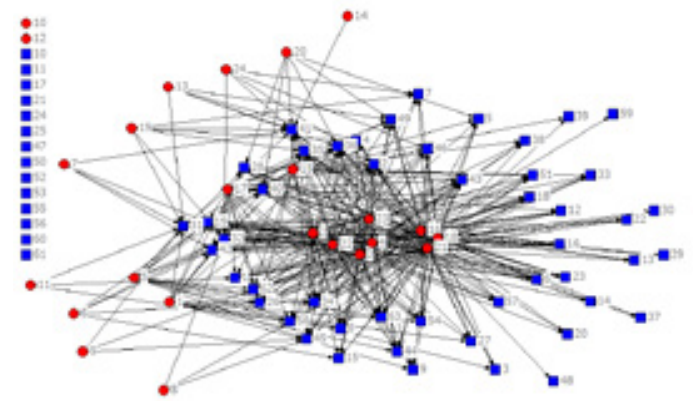

Fig. 5. Two-mode network for medium firms with ideas from foreign sources.

Medium enterprises receiving ideas from national sources (Fig. 6) shows higher centrality for the following national sources: 4 (154), 5 (155), 6 (156), 7 (157), 13 (173), 14 (174), 15 (175), 16 (181), 21 (203), 23 (221), and 24 (222).

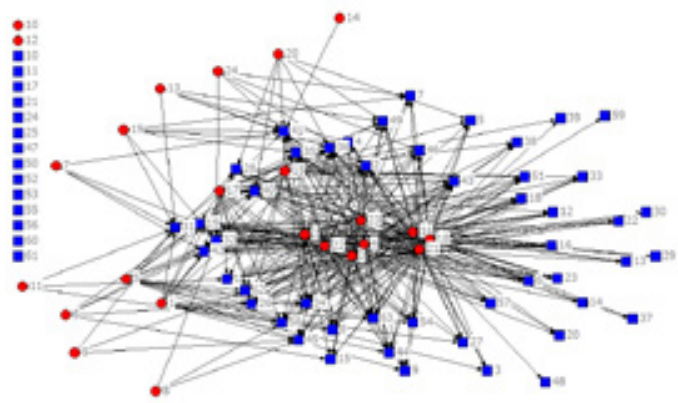

Fig. 6. Two-mode network for medium firms with ideas from national sources.

Small firms reveals a structure of the network where main foreign sources of ideas to produce innovations (Fig. 7) are: 3 (153), 4 (154), 16 (181), 18 (191), 21 (201), and 23 (203).

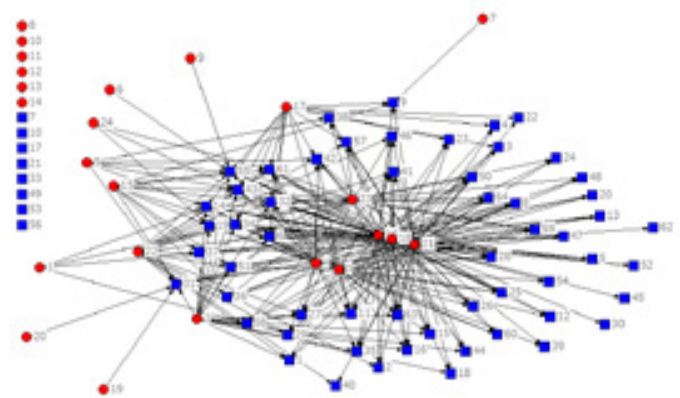

Fig. 7. Two-mode network for small firms with ideas from foreign sources.

When examining small firms linked to their national sources of ideas (Fig. 8) the results are: 2 (152), 7 (157), 16 (181), 17 (182), 18 (191), 21 (201), 23 (203), and 24 (204).

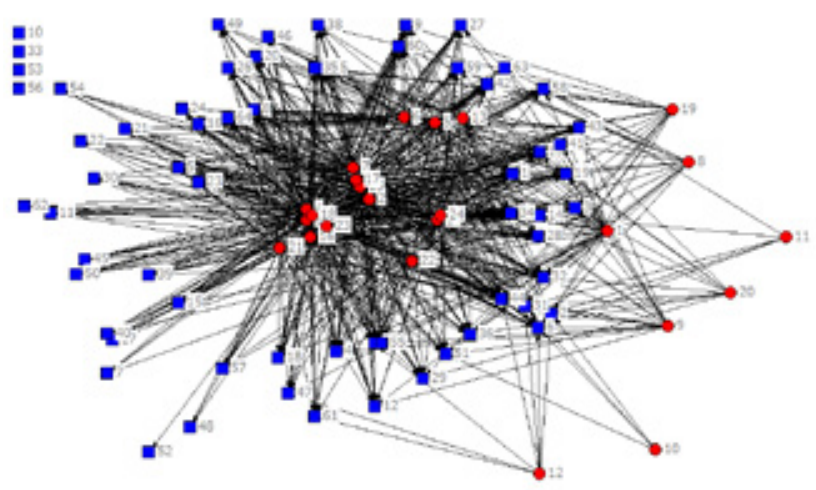

Fig. 8. Two-mode network for small firms with ideas from national sources.

When applying the projection operator over the two-mode network of large firms - foreign sources of ideas (Fig. 3) results that main sources of ideas to generate innovation (Fig. 9) are: 2 (Scientific and technological databases), 16 (Internet), 17 (Technical regulations and standards), and 19 (technological parks) (Table II). 


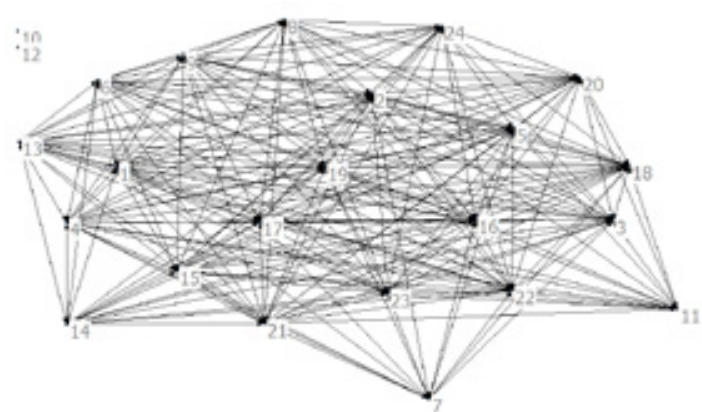

Fig. 9. One-mode network (obtained by projection) for large firms with ideas from foreign sources. The dataset of projection corresponds to the twenty four sources of ideas listed in Table II.

Projection of the two-mode network (Fig. 4) of large firms recipients of ideas from national sources results in a one-mode network (Fig. 10) lets identify the higher centrality nodes, that reveal that main national sources are: 12 (firms from a different sector), 13 (Trade fairs and exhibitions), 14 (Technology-based companies and incubators), 21 (Seminars and conferences) y 23 (industrial property information systems).

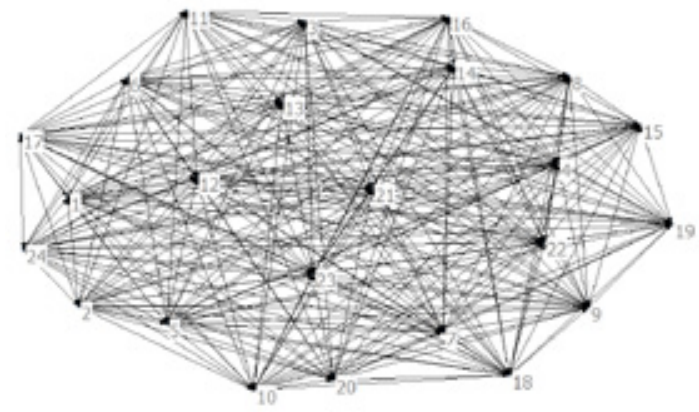

Fig. 10. One-mode network (obtained by projection) for large firms with ideas from national sources. The dataset of projection corresponds to the twenty four sources of ideas listed in Table II.

Projection of the two-mode network (Fig. 5) of medium firms recipient of ideas from foreign sources, in a one-mode network (Fig. 11) reveals that foreign sources: 10 (Consultants or experts) and 12 (Firms form other sectors) are not relevant. In contrast, the rest of foreign sources are probabilistically equal sources of ideas for innovation.

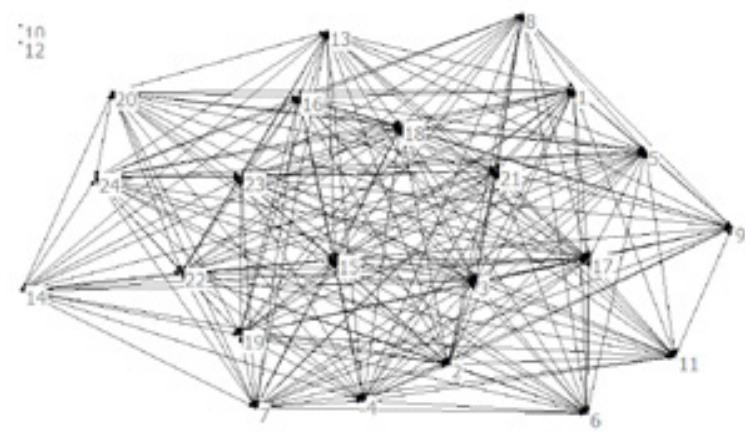

Fig. 11. One-mode network (obtained by projection) for medium firms with ideas from foreign sources. The dataset of projection corresponds to the twenty four sources of ideas listed in Table II.

Projection of the two-mode network (Fig. 6) of medium firms recipient of ideas from national sources, in a one-mode network (Fig. 12) shows that main sources of ideas for innovation are: 15 (Public institutions), 16 (Internet), 17 (Books, journals and catalogs), 18 (Technical regulations and standards), 19 (Technological parks), and y 21 (seminars and conferences).

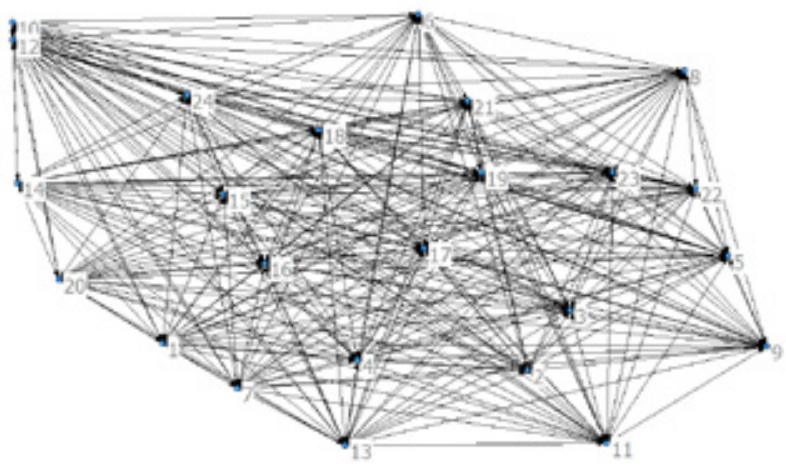

Fig. 12. One-mode network (obtained by projection) for medium firms with ideas from national sources. The dataset of projection corresponds to the twenty four sources of ideas listed in Table II.

The case for small firms, when projecting their two-mode network (Fig. 7), results in a one-mode network (Fig. 13) showing that main foreign sources of ideas for innovation are: 2 (scientific and technological databases), 4 (Technological development centers), 16 (Internet), and 21 (seminars and conferences).

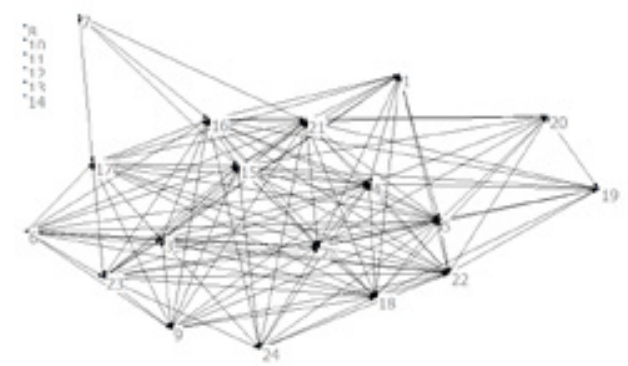

Fig. 13. One-mode network (obtained by projection) for small firms with ideas from foreign sources. The dataset of projection corresponds to the twenty four sources of ideas listed in Table II.

Projection of the two-mode network (Fig. 8) of small firms recipient of ideas from national sources, in a one-mode network (Fig. 14) shows that main sources of ideas for innovation are: 2 (scientific and technological databases), 3 (Chambers of Commerce), 4 (Technological development centers), 15 Public institutions, 16 (Internet), 17 (Books, journals and catalogs), and 21 (Seminars y conferences).

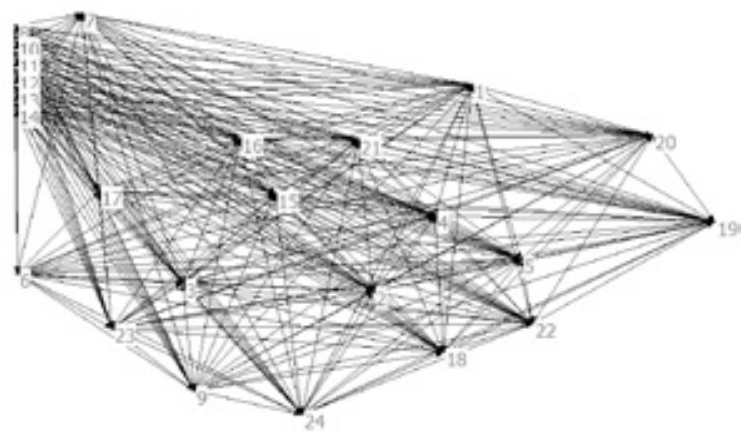

Fig. 14. One-mode network (obtained by projection) for small firms with ideas from national sources. The dataset of projection corresponds to the twenty four sources of ideas listed in Table II. 


\section{DISCUSSION}

From the analysis, the firms from the manufacturing sub-sector, obtaining ideas to innovate from foreign sources are (ISIC Rev. 3. A. C.): 151 Production, processing and preserving of meat and meat products, 152 Processing and preserving of fruits, legumes, green vegetables, oil and fat, 153 Manufacture of fresh liquid milk, pasteurized, sterilized, homogenized and/or other dairy products, 154 Manufacture of starches and starch products, 175 Manufacturing of fabrics and knitting fabrics, 181 Manufacturing of clothing, except leather based, 191 Leather products, 192 Leather-shoe manufacturing, 203 Manufacture of wooden goods intended to be used primarily in the construction industry, 210 Manufacture of paper and board and paper and board products, and 221 Publishing, printing and reproduction of recorded media.

In addition, main foreign sources of ideas for large firms from those subsectors are scientific and technological databases, technological parks, clients and consultants or experts.

Those firms of subsector 221 Publishing, printing and reproduction of recorded media, prefer to get ideas from international consultants an experts. They complement their knowledge by means of ideas coming from foreign technological parks and information from scientific and technological databases, the specifications received from their international clients.

Ideas coming from technological parks and international consultants for enterprises of 151 Production, processing and preserving of meat and meat products and 153 Manufacture of fresh liquid milk, pasteurized, sterilized, homogenized and/or other dairy products, may allow them the ability to develop preventive actions such as improving competitiveness or even develop competitive advantages- to face foreign competitors in the context of free trade agreements.

However, it seems that the identified sources of ideas for innovation in large firms of the manufacturing sector served to adopt short-term, minimum national competence positions.

A better comprehension of the relationships between the sources of ideas for innovation, according to its origin (foreign or national), and manufacturing firms may provide background to develop policies to encourage firms to adopt an active leading position. Nevertheless, with improved knowledge on the structure of innovation in the manufacturing sector, to strengthen the use of foreign sources of ideas for innovation such as scientific and technological databases, and include the gain of contracting consultants from local universities that may understand local markets better than foreign consultants.

\section{CONCLUSION}

A simple but powerful methodology to investigate the structure of innovation, from SITD and SITDS, based in knowledge transfer in interfirm contexts was developed. This methodology is well founded in social network analysis and easy to use information technology tools.

It was shown that this methodology facilitates a structured generation of information related to the links between sources and receivers of ideas for innovation, allowing to identify preferred sources by firm size, according to the origin of the source (foreign or national).

The methodology that has been introduced and demonstrated in use, satisfies the aim to serve as methodological reference to identify sources of ideas in knowledge transfer to be effectively used in innovation.

Codification of dimensions when constructing datasets may generate biases. In spite of this fact, the methodology remains effective because the relevant findings is on the network structure (identified patterns on the main sources of ideas to generate innovation by any set of firms in ISIC).
These methodology is of interest for foreign companies that may find in Colombia the destination of their products, general activities, and consultancy contracts opportunities.

Finally, in order to compare results from different research processes, a unified but simple methodology facilitates researchers to concentrate on the field of investigation and not in the development of an ad-hoc methodology or the time-consuming learning of a complicated, even complete, methodology.

\section{FURTHER WORK}

This work reports an analysis on two-mode networks emerging from the relations between national and foreign sources of ideas for innovation and manufacturing firms, based on indegree calculation. Additional work may include determining if other centrality and prestige measures may provide accurate information on sources of ideas.

To enhance this methodology, in terms of informing data to stakeholders, a short-term economic oriented analysis may be included. This short-term analysis should include information on international free trade agreements and a transition analysis of foreign commerce policies.

\section{ACKNOWLEDGMENT}

Authors express their gratitude to Prof. Rafael Hurtado, at Universidad Nacional de Colombia, for providing useful counselling during the development of this research project. This paper is based on the presentation at CONIITI 2015.

\section{REFERENCES}

[1] J. H. Dyer and N. W. Hatch, "Network-specific capabilities, network barriers to knoledge transfers, and competitive advantage," in Academy of Management Proceedings, 2004, pp. V1-V6.

[2] R. M. Grant, "The knowledge-based view of the firm: implications for management practice," Long range planning, vol. 30, pp. 450-454, 1997.

[3] M. Boisot and A. Canals, "Data, information and knowledge: have we got it right?," Journal of Evolutionary Economics, vol. 14, pp. 43-67, 2004.

[4] C. E. Shannon and W. Weaver, The mathematical theory of information. Urbana, Illinois.: University of Illinois Press, 1949.

[5] P. R. Carlile, "Transferring, translating and transforming: an integrative relational approach to sharing and assessing knowledge across boundaries," Organization Science, vol. 15, pp. 555-68, 2004.

[6] J. E. Arias Pérez and C. A. Aristizábal Botero, "El dato, la información, el conocimiento y su productividad en empresas del sector público de Medellín,” Semestre Económico, vol. 14, pp. 95-109, 2011.

[7] G. Durant-Law, "TARDIS: A Journey Through an Enterprise Knowledge Space," Master of Knowledge Management, School of Information and Management, University of Canberrra, Canberra, Australia [http:// www.durantlaw.info/sites/durantlaw.info/files/TARDIS Final Document.pdf], 2004.

[8] R. McDermott, "Why information technology inspired but cannot deliver knowledge management," Knowledge and communities, vol. 41, pp. 21$35,2000$.

[9] G. Dosi, C. Freeman, and S. Fabiani, "The process of economic development: introducing some stylized facts and theories on technologies, firms and institutions," Industrial and Corporate Change, vol. 3, pp. 1-45, 1994.

[10] E. E. Hagen, "The process of economic development," Economic Development and Cultural Change, pp. 193-215, 1957.

[11] [11] I. Adelman, Theories of economic growth and development. Stanford, Calif.: Stanford University Press, 1961.

[12] R. J. Barro, X. Sala-i-Martin, and M. I. T. Press, Economic growth. Cambridge; London: The MIT Press, 1999. 
Regular Issue

[13] N. Kondratieff and W. Stolper, "The Long Waves in Economic Life," The Review of Economics and Statistics, vol. 17, pp. 105-115, Nov. 19351935.

[14] M. Avella and L. Fergusson, "El ciclo económico, enfoques e ilustraciones. Los ciclos económicos de Estados Unidos y Colombia," Borradores de Economía, vol. 284, pp. 1-78, 2003.

[15] R. H. Day, "Irregular growth cycles," The American Economic Review, pp. 406-414, 1982.

[16] N. Khabiri, S. Rast, and A. A. Senin, "Identifying main influential elements in technology transfer process: a conceptual model," Procedia-Social and Behavioral Sciences, vol. 40, pp. 417-423, 2012.

[17] V. Gilsing, R. Bekkers, I. M. B. Freitas, and M. van der Steen, "Differences in technology transfer between science-based and development-based industries: Transfer mechanisms and barriers," Technovation, vol. 31, pp. 638-647, 2011.

[18] UNCTAD, Transfer of technology. New York, 2001.

[19] E. M. Rogers, Diffusion of innovations, 5th ed. New York. UJ303.484 R64 2003 Colección alterna No.2: Free Press. N.Y., 2003.

[20] A. Hevner and S. Chatterjee, Design research in information systems: theory and practice vol. 22: Springer Science \& Business Media, 2010.

[21] E. T. Layton Jr, "Technology as knowledge," Technology and culture, pp. 31-41, 1974.

[22] K. J. Arrow, "Classificatory notes on the production and transmission of technological knowledge," The American Economic Review, pp. 29-35, 1969.

[23] E. M. Rogers, "The nature of technology transfer," Science Communication, vol. 23, pp. 323-341, 2002.

[24] E. G. Carayannis, "Knowledge transfer through technological hyperlearning in five industries," Technovation, vol. 19, pp. 141-161, 1999.

[25] G. Von Krogh, K. Ichijo, and I. Nonaka, Enabling knowledge creation: How to unlock the mystery of tacit knowledge and release the power of innovation: Oxford university press, 2000.

[26] G. Von Krogh, "Understanding the problem of knowledge sharing," International journal of information technology and management, vol. 2, pp. 173-183, 2003.

[27] A. Wahab, A. Sazali, H. Uli, and R. Rose, "Evolution and development of technology transfer models and the influence of knowledge-based view and organizational learning on technology transfer," Research Journal of International Studies, vol. 12, pp. 79-91, 2009.

[28] D. V. Gibson and R. W. Smilor, "Key variables in technology transfer: A field-study based empirical analysis," Journal of Engineering and Technology management, vol. 8, pp. 287-312, 1991.

[29] T. K. Sung and D. V. Gibson, "Knowledge and technology transfer: levels and key factors," in Proceeding of the 4th International Conference on Technology Policy and Innovation, 2000, pp. 3-7.

[30] E. S. Rebentisch and M. Ferretti, "A knowledge asset-based view of technology transfer in international joint ventures," Journal of Engineering and Technology Management, vol. 12, pp. 1-25, 1995.

[31] S. Abdul Wahab, R. C. Rose, U. Jegak, and H. Abdullah, "A review on the technology transfer models, knowledge-based and organizational learning models on technology transfer," European journal of social sciences, vol. 10, 2009.

[32] S. M. Wagner and C. Buko, "An empirical investigation of knowledgesharing in networks," Journal of Supply Chain Management, vol. 41, pp. 17-31, 2005.

[33] G. P. Huber, "Organizational learning: The contributing processes and the literatures," Organization science, vol. 2, pp. 88-115, 1991.

[34] I. Nonaka and H. Takeuchi, The knowledge-creating company : how Japanese companies create the dynamics of innovation. New York [etc.]: Oxford University Press, 1995.

[35] I. Nonaka, R. Toyama, and A. Nagata, "A firm as a knowledge-creating entity: a new perspective on the theory of the firm," Industrial and corporate change, vol. 9, pp. 1-20, 2000.

[36] R. M. Grant, "Toward a knowledge-based theory of the firm," Strategic management journal, vol. 17, pp. 109-122, 1996.

[37] C. Dhanaraj, M. A. Lyles, H. K. Steensma, and L. Tihanyi, "Managing tacit and explicit knowledge transfer in IJVs: the role of relational embeddedness and the impact on performance," Journal of International Business Studies, vol. 35, pp. 428-442, 2004.

[38] J. L. Moreno, "Sociogram and sociomatrix," Sociometry, vol. 9, pp. 348-
$349,1946$.

[39] J. L. Moreno, "Sociometry in relation to other social sciences," Sociometry, vol. 1, pp. 206-219, 1937.

[40] S. Wasserman, Social network analysis: Methods and applications vol. 8: Cambridge university press, 1994.

[41] M. E. J. Newman, Networks : an introduction. Oxford; New York: Oxford University Press, 2010.

[42] J. Park and M. E. Newman, "The statistical mechanics of networks," Phys. Rev. $E$ vol. 70 p. 066117 doi:10.1103/PhysRevE.70.066117, 2004.

[43] M. E. Newman, "The structure and function of complex networks," SIAM review, vol. 45, pp. 167-256 2003 ( available at arXiv.org/condmat/0303516).

[44] H. A. Simon, "On a class of skew distribution functions," Biometrika, pp. 425-440, 1955.

[45] R. G. Hurtado and J. E. Mejía, "The structure of investment for technological innovation and development activities in the colombian manufacturing industry," Innovar, vol. 24, pp. 33-40, 2014.

[46] J. C. Duque, S. J. Rey, and D. A. Gómez, "Identifying industry clusters in Colombia based on graph theory," Ensayos Sobre Politica Económica, vol. 27, pp. 14-45, 2009.

[47] DMReview. (2007). Glossary. SourceMedia (available at www. dmreview.com/glossary/a.html).

[48] N. Prakash, "A process view of methodologies," in Advanced Information Systems Engineering, 1994, pp. 339-352.

[49] R. Veryard, "What are methodologies good for?," Data Processing, vol. 27, pp. 9-12, 1985.

[50] . Bussmann, R. Nicholas, and M. Wooldridge, "On the identification of agents in the design of production control systems," in Agent-Oriented Software Engineering, 2001, pp. 141-162.

[51] G. Pólya, Mathematical discovery; on understanding, learning, and teaching problem solving (1981, Combined edition). New York: John Wiley \& Sons, 1962,

[52] V. H. Medina García, S. BolañosCastro, and R. González Crespo, "Process Management Software as a Script, and the Script as a Pattern," Interbnational Journal of computer and Communication Engineering vol. 1, pp. 147-150, 2012.

[53] S. J. Bolaños Castro, R. González Crespo, and V. H. Medina García, "Antipatterns: a compendium of bad practices in software development processes," IJIMAI, vol. 1, pp. 41-46, 2011.

[54] S. J. Bolaños Castro, R. González Crespo, and V. H. Medina García, "Patterns of software development process," IJIMAI, vol. 1, pp. 33-40, 2011.

[55] Pacheco, A., H. Bolivar-Baron, R. Gonzalez-Crespo, and J. PascualEspada, "Reconstruction of High Resolution 3D Objects from Incomplete Images and 3D Information", International Journal of Interactive Multimedia and Artificial Intelligence, vol. 2, issue Regular Issue, no. 6, pp. 7-16,06/2014

[56] S. P. Borgatti, M. G. Everett, and L. C. Freeman, "Ucinet for Windows: Software for social network analysis," 2002.

[57] S. P. Borgatti, "NetDraw software for network visualization," Lexington, KY: Analytic Technologies, p. 95, 2002.

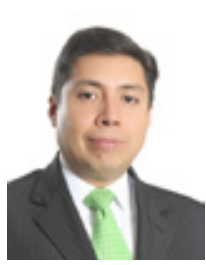

Orlando López-Cruz is member of the research group "Riesgo en sistemas naturales y antrópicos", Ph.D.(c) in Engineering, Pontifical Xaverian University, Master in Administration, Universidad Nacional de Colombia, Organizational Control Systems Specialist, Universidad de los Andes, Colombia, Economist, Central University, Colombia. He has held management positions at Organización Sanitas Internacional (Colsanitas), Colsubsidio, and consultant at Gesfor Group. He held teaching positions at Universidad del Rosario, Bogotá, and Central University, Bogotá. Currently, he is associate professor at Universidad El Bosque, Bogotá. Prof. López-Cruz research and consulting interests include Processes and structures design for innovations in organizations, organizational knowledge transfer, engineering complex controls for organizations, design and implementation of agent-based systems for social systems, and adequate implementation of organizational information systems. 


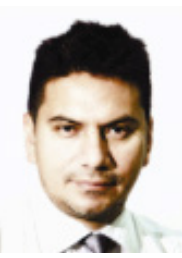

Nelson Obregón Neira is member of the research group "Riesgo en sistemas naturales y antrópicos", Ph.D. in Hydrology, University of California, Davis, U.S.A. Master in Civil Engineering, Universidad de Los Andes, Bogotá. Civil Engineer, Universidad Francisco de Paula Santander, Cúcuta, Colombia.He has held teaching and research positions at Pontifical Xaverian University and Universidad Nacional de Colombia. He currently is Director of Instituto Geofisico. Prof. Obregón Neira research and consulting interests include: Intelligent Technologies, turbulent processes, Chaos theory, applied fractal geometry, Data mining and Learning Machine in Geosciences. Computational modeling and applied mathematics (deterministic, statistical and probabilistic mathematics). 